\title{
Development of tunnel compression wave generator with multiple small solenoid valves
}

\author{
Tokuzo MIYACHI*, Takakage ARAI**, Shoji SAKAUE**, Koji TAKASHIMA**, Daiki TANIKAWA**, \\ Takuya YAGISHITA** and Kento NAKATANI** \\ * Railway Technical Research Institute \\ 2-8-38 Hikari-cho, Kokubunji, Tokyo 185-8540, Japan \\ E-mail: miyachi.tokuzo.06@rtri.or.jp \\ ** Osaka Prefecture University \\ 1-1 Gakuen-cho, Naka-ku, Sakai, Osaka 599-8531, Japan
}

Received: 22 October 2018; Revised: 13 December 2018; Accepted: 7 February 2019

\begin{abstract}
The micro-pressure wave (MPW) phenomenon caused by a high-speed train entering a tunnel consists of three stages: generation of the tunnel compression wave upon train entry, propagation of the tunnel compression wave through the tunnel, and emission of the MPW outward. Model experiments using train launcher facilities (TLFs) are effective for analyzing MPWs. However, the use of those facilities to study the latter two stages, i.e., the compression wave propagation and MPW emission, is hindered by two problems: high costs required for facility operation and construction, and low data productivity. Compression wave generators can potentially provide reasonable experiments. In this study, a new, simple facility was developed for the simulation of tunnel compression waves. The proposed generator consists of small solenoid valves, a pressure chamber, compressor, power supply system, and tunnel model. The performance analyses indicate that the compression waves generated by the designed facility effectively simulate tunnel compression waves generated by train entry. The pressure rise and wavelength of the compression wave are controlled by adjusting the chamber pressure and the voltage applied to the valves. The generator is expected to provide sufficient data to develop measures against MPWs in the propagation and emission stages as it reduces the required time by over 60\% compared to that required by TLFs for each generation of the compression waves.
\end{abstract}

Keywords : High-speed train, Micro-pressure wave, Experimental facility, Shock tube, Tunnel compression wave

\section{Introduction}

The micro-pressure waves (MPWs) (Yamamoto (1977), Ozawa (1979)) emitted from railway tunnels are one of the environmental problems caused by high-speed trains. As MPWs can generate noise and vibration, various studies have been conducted to mitigate them. Model experiments are effective for studying MPWs, and experimental facilities that can launch a train model at 200-500 km/h are in use worldwide (Johnson and Dalley (2002), Heine and Ehrenfried (2012), Fukuda (2013), Zhou et al. (2014), Yang et al. (2016)). At the Railway Technical Research Institute (RTRI) in Japan, the first train-launcher facility (TLF) was developed in the late 1950s for the technical preparation of the first high-speed train, Shinkansen (Fukuda (2013)). TLFs have provided increased maximum launch speeds in accordance with increases in the Shinkansen speed. Today, the seventh facility at RTRI can launch a train model at $550 \mathrm{~km} / \mathrm{h}$ (Fukuda (2013)). Owing to research conducted using these facilities, the Shinkansen is operated at $320 \mathrm{~km} / \mathrm{h}$ without serious MPW problems.

When a high-speed train enters a tunnel, a compression wave, called "tunnel compression wave" in this paper, is generated. This tunnel compression wave propagates through the tunnel towards the exit at the speed of sound. The tunnel compression wave is then reflected at the exit and returns to the entrance. Upon reflection, an MPW is emitted outside the tunnel (Ozawa (1979)). Hence, the MPW phenomenon can be divided into three stages: (a) generation of the tunnel compression wave, (b) propagation of the tunnel compression wave, and (c) emission of the MPW. The TLFs 


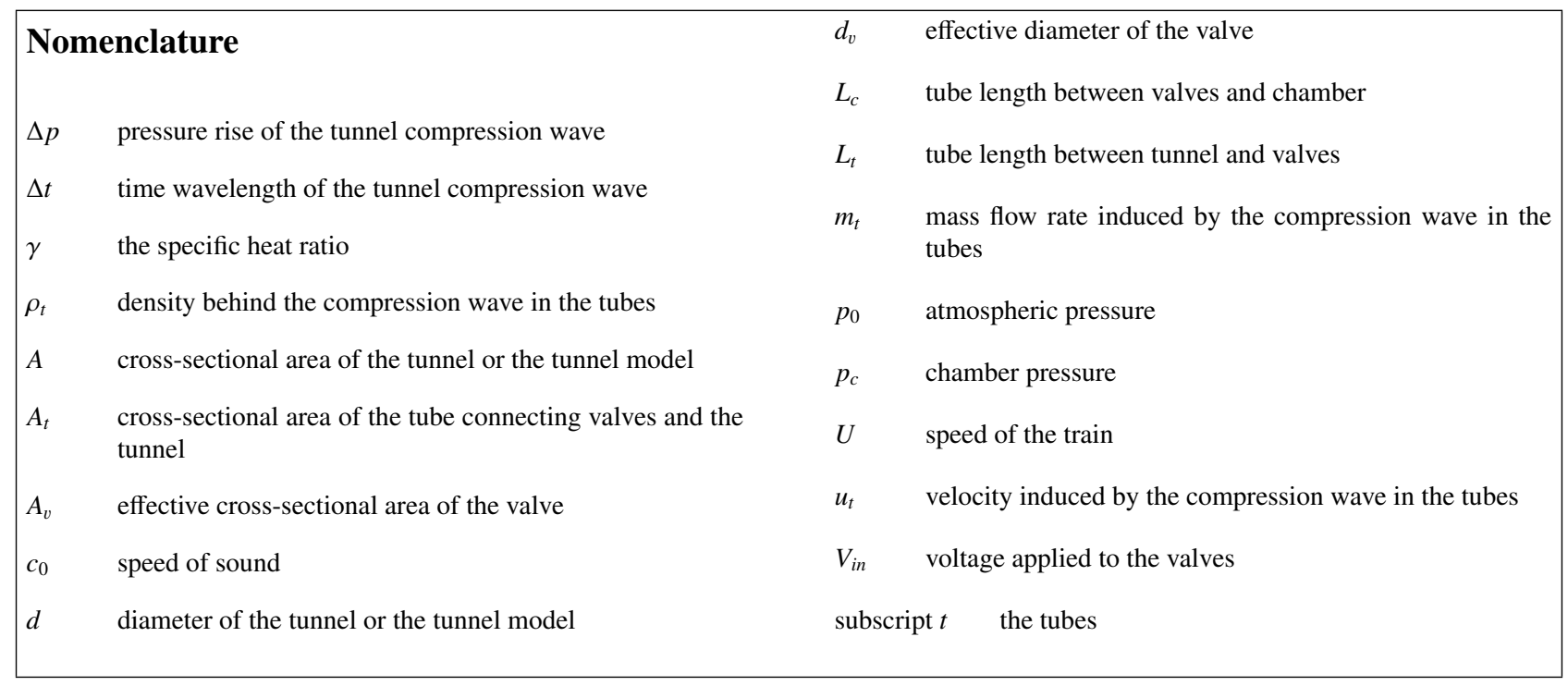

are effective with respect to model experiments for stage (a), in which the influence of the train nose shape and tunnelentrance configuration are examined (Ozawa (1979), Iida et al. (1996)). In stage (b), the primary interest is in the steepening of the compression wave (Miyachi et al. (2016)). The steepening depends on the nonlinear effect of the fluid, boundary layer, tunnel configuration, etc. To attenuate the steepening, the influence of ballast, branches, resonators, and absorbers are studied (Ozawa et al. (1997), Vardy and Brown (2000), Miyachi et al. (2014), Tebbutt et al. (2017)). In stage (c), the influences of the exit hood and topography of the ground near the exit portal have been studied (Miyachi and Fukuda (2014), Miyachi (2016)). However, as only few studies have investigated the phenomena in stages (b) and (c) with model facilities, good countermeasures to MPWs in these stages have not been developed yet. To increase the maximum train speed, model experiments on these stages are of utmost importance as the magnitude of MPWs is proportional to $U^{n}$, where $U$ is the train speed and $n>3$ for slab track tunnels. For such experiments, the tunnel compression wave generation due to train entry does not need to be studied using TLFs. Instead, we can investigate the phenomena on stages (b) and (c) by using compression waves with wavelengths and magnitudes similar to those of the tunnel compression waves.

As the construction of TLFs is very expensive, not many researchers use them. When constructing a new TLF, we need not only enough budget but also a considerable amount of space and human resources to operate and maintain the facility. In addition, data productivity of the TLFs is not very high because many procedures are employed for the generation of a compression wave. If we can easily generate compression waves by using affordable facilities compared to TLFs, more experiments at a more accelerated pace can be conducted on MPWs in stages (b) and (c).

Experimental facilities that generate compression and/or shock waves have been developed for various purposes. In this paper, such facilities generating compression waves are called "compression wave generators." In a broad sense, TLFs are also a type of compression wave generators. For example, Sugimoto et al. (2014) used a compression wave generator using a piston driven by high-pressure air for conducting experiments on soliton waves in air. Shock tubes (Martin (1959)) have also been used effectively for shock-wave research, and they are simple and practical devices compared to TLFs in terms of construction costs, as they usually consist only of high- and low-pressure rooms separated by a diaphragm.

However, the use of shock tubes to generate tunnel compression waves appears challenging. For instance, the pressure range and time scale required for tunnel compression wave generators (TCGs) are very small and large, respectively, compared to those for conventional shock tubes using a diaphragm. If a high-speed solenoid valve is used instead of a diaphragm, a very high-pressure chamber is needed to generate the required pressure rise in the tunnel model, as such valves have small effective cross-sectional areas. Consequently, the development of unusual valves or a simple solution is required as we cannot solve this problem by using commercial valves employed conventionally. To develop a TCG, Matsuo et al. (1991) developed a rapid-opening valve for a pipe with an approximate diameter of $20 \mathrm{~mm}$, successfully generating compression waves with time wavelengths of a few milliseconds.

In this study, we developed a new, simple compression wave generator with multiple small solenoid valves. The generator is affordable and very compact, and it has high data productivity compared with that of TLFs. 


\section{TCG}

\subsection{Tunnel compression wave}

The pressure rise of a tunnel compression wave, $\Delta p$, generated by a train entering a tunnel, is expressed as (Hara (1961))

$$
\Delta p=\frac{1}{2} \rho U^{2} \frac{1-(1-R)^{2}}{(1-M)\left(M+(1-R)^{2}\right)},
$$

where $\rho$ is the atmospheric density, $U$ is the train speed, $M$ is the train Mach number, and $R$ is the blockage ratio of train to tunnel. For a Shinkansen, as $U \sim 300 \mathrm{~km} / \mathrm{h}$ and $R \sim 0.2, \Delta p \sim 2 \mathrm{kPa}$.

To avoid serious noise problem of the MPW, the pressure gradient of the compression wave is required to be less than $10-20 \mathrm{kPa} / \mathrm{s}$. Thus, tunnel hoods are installed at the tunnel entrance to reduce the pressure gradient to this target value. Figure 1 shows the typical pressure gradient waveforms of the tunnel compression waves measured using a 1:127 scale model of a TLF. Pressure rise $\Delta p \approx 3 \mathrm{kPa}$, and the pressure gradient is reduced from 3 to $1 \mathrm{MPa} / \mathrm{s}$, which roughly corresponds to 30 and $10 \mathrm{kPa} / \mathrm{s}$, respectively, because of the use of a hood. While the pressure gradient waveform has a single peak when no hood is present, it has multiple peaks when a hood exists (Ozawa (1979), Miyachi et al. (2016)).

without a hood

with a hood

Train launcher facility at $360 \mathrm{~km} / \mathrm{h}, 1 / 127$ scale model test

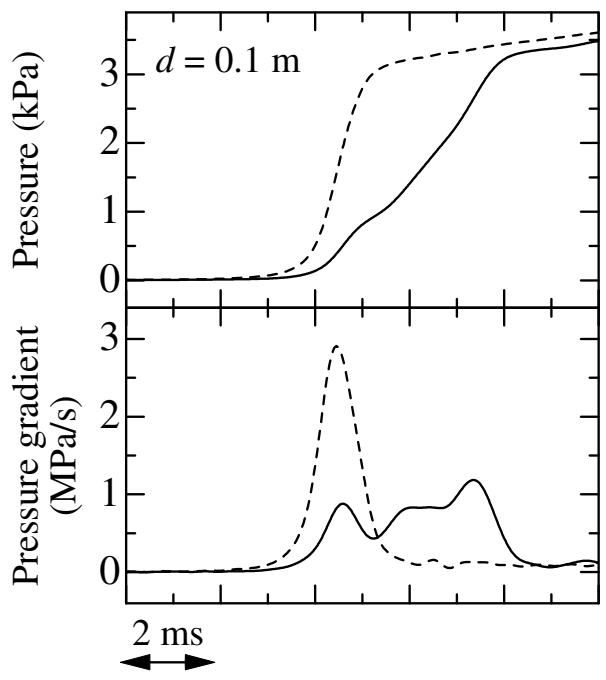

Fig. 1 Typical waveforms of tunnel compression waves

\subsection{Basic design of a TCG}

The main purpose of a TCG is not to simulate the relationship between the shapes of train noses and tunnel compression waves but to generate a compression wave similar to representative tunnel compression waves. These representative waves have an approximate pressure rise of $\Delta p=2 \mathrm{kPa}$ at the tunnel entrance, with a maximum pressure gradient of $10-20 \mathrm{kPa} / \mathrm{s}$ and $\Delta t=0.1-0.2 \mathrm{~s}$ in full scale, where $\Delta t$ is the time wavelength of the compression wave for $300 \mathrm{~km} / \mathrm{h}$ Shinkansen trains. When a $\phi 100-\mathrm{mm}$ pipe is used as a tunnel model, its scale relative to the Shinkansen tunnels is approximately $1 / 100$. As the cross-sectional area of the Shinkansen tunnel is approximately $60 \mathrm{~m}^{2}$, the target TCG values developed here are $\Delta p=2 \mathrm{kPa}$, and the maximum pressure gradient is $1-2 \mathrm{MPa} / \mathrm{s}$. The time scale must satisfy $\Delta t \sim 1 \mathrm{~ms}$. When valves instead of shock-tube diaphragms are employed, Boyle's law roughly estimates that $\Delta p / p_{c} \sim d_{v}^{2} / d^{2}$, where $p_{c}$ is the chamber pressure, $d_{v}$ is the effective diameter of the valve, $d$ is the Shinkansen tunnel diameter, and $A_{v}$ is the effective cross-sectional area of the valve $\left(A_{v}=\pi d_{v}^{2} / 4\right)$. Although small valves have rapid responses ( $(\mathrm{small} \Delta t)$, as $d_{v}$ is excessively small, the value of $p_{c}$ required for obtaining sufficient $\Delta p$ is very large. Therefore, the mass flow rate through the orifice in the solenoid valve is extremely small, with conventional chambers that have insufficient air tightness at such a large value of $p_{c}$. Even for conventionally large electric valves, $A_{v} \sim 500 \mathrm{~mm}^{2}$ and $\Delta t \sim 100 \mathrm{~ms}$; thus, $\Delta t$ is very large for a $1 / 100$-scale model test. 
In the proposed design, to overcome the problem of insufficient mass flow rate for small high-speed solenoid valves, multiple solenoid valves are used in parallel. We used commercial and reasonable valves in the generator. Figure 2 shows the schematic of the TCG developed in this study and Figure 3 shows photographs of the TCG and valves. Ten tubes with inner diameters of $8 \mathrm{~mm}$ connect the pressure chamber and tunnel. Each tube has a valve, and each of the compression waves generated by each solenoid valve propagates through each of the tubes and flange before reaching the tunnel model. Although the TCG uses a small amount of air in the chamber for testing, as the measurement is stopped before any waves from the chamber reach the test section (see Sec. 3.1), the volume should be sufficiently large to easily control the chamber pressure at a target value. The tunnel is made of polyvinyl chloride and has a diameter of $100 \mathrm{~mm}$ and a scale of $1 / 127$ with respect to the mirror image of the tunnel.

The TCG is required to control $\Delta p$ and $\Delta t$ of the compression wave to simulate the changes in the tunnel compression waves related to the cross-sectional area and speed of the train. For the TCG developed in this study, $\Delta p$ and $\Delta t$ can easily be controlled by adjusting the chamber pressure and valve-opening speed, respectively. However, the TCG requires trial and error to control multiple-peak pressure gradient waveforms.

For the valves in the generator, the electromagnetic force and opening time change according to the applied voltage. Therefore, we developed a power-supply device consisting of batteries, a photo metal-oxide-semiconductor relay (Panasonic AQZ262), variable resistor, and trigger. Voltage $V_{\text {in }}$ applied to the solenoid valves can be adjusted using the variable resistor and/or by adjusting the number of batteries. To prevent abnormal overheating of the solenoid valves, a timer is used in the power-supply device. The timer causes the circuit to automatically deactivate after a certain period following the input trigger signal. As a result, the valves are closed within $1 \mathrm{~s}$. Although the electric-power consumption of the valves is small, we need $100 \mathrm{~A}$ with $10 \mathrm{~V}$ to simultaneously open all valves because a large current pulse of approximately $10 \mathrm{~A}$ with $10 \mathrm{~V}$ is required to open each valve. To minimise the expenditure incurred to solve this problem, commercial batteries for conventional cars were used as the DC power source.

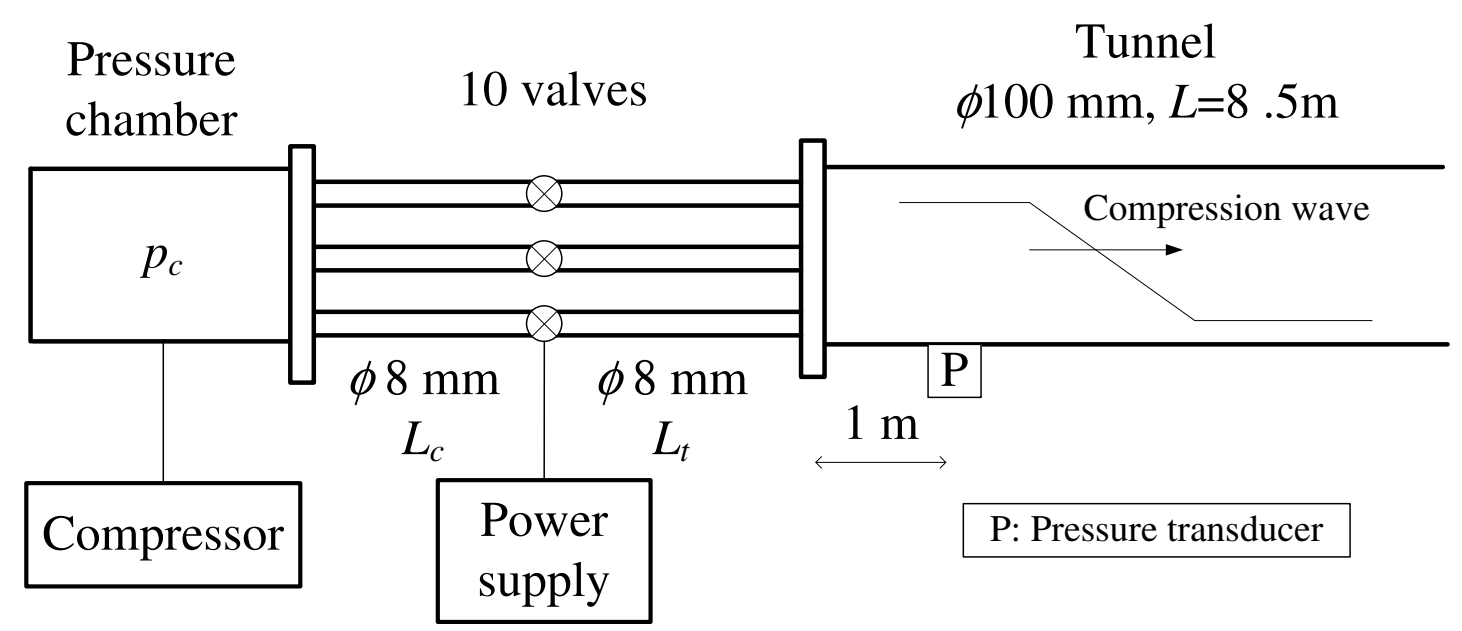

Fig. 2 Schematic of the tunnel compression wave generator

\section{Results}

\section{1. $x-t$ diagram}

Figure 4 illustrates compression waveforms obtained $1 \mathrm{~m}$ from the flange, where $(\mathrm{G})$ denotes gauge pressure. The first pressure rise is caused by the first compression wave, which simulates the tunnel compression wave. Subsequent pressure fluctuations are also apparent. These fluctuations are explained as follows.

Fig. 5 shows two $x-t$ diagrams for the different lengths of tubes between the valves and tunnel $\left(L_{t}\right)$ and between the valves and pressure chamber $\left(L_{c}\right)$, as presented in Fig. 2. When the solenoid valves open, compression wave XC1 and expansion wave YE1 are generated. Part of YE1 is reflected as compression wave YC1 at the junction of the chamber. Then, YC1 propagates through the solenoid valves and reaches the measurement section. However, the orifice is small; thus, the influence of $\mathrm{YC} 1$ is almost negligible. XC1 reflects at the end of the tunnel as expansion wave XE1, which then reflects at the flange as XE2. Part of XC1 reflects at the flange and propagates in the direction of the solenoid valves as expansion wave ZE1. Then, almost all of ZE1 reflects at the orifice of the solenoid valves and reaches the measurement point as expansion wave ZE2. The measurement period for the tunnel compression wave is between the arrivals of XC1 


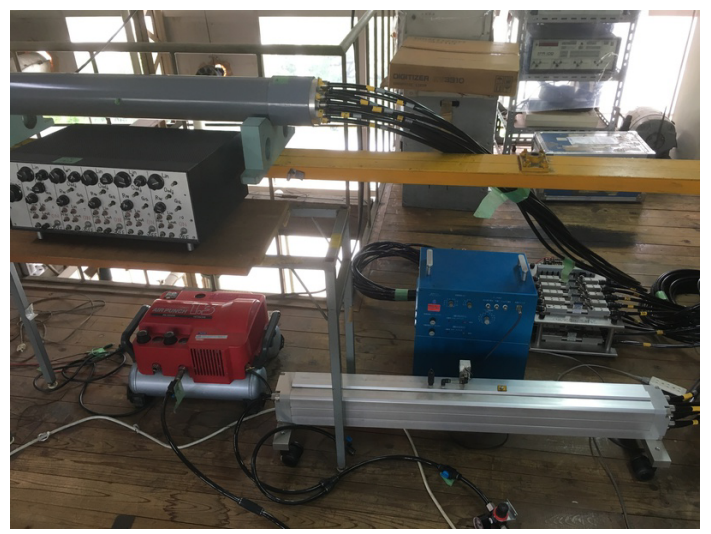

(a) Compression wave generator

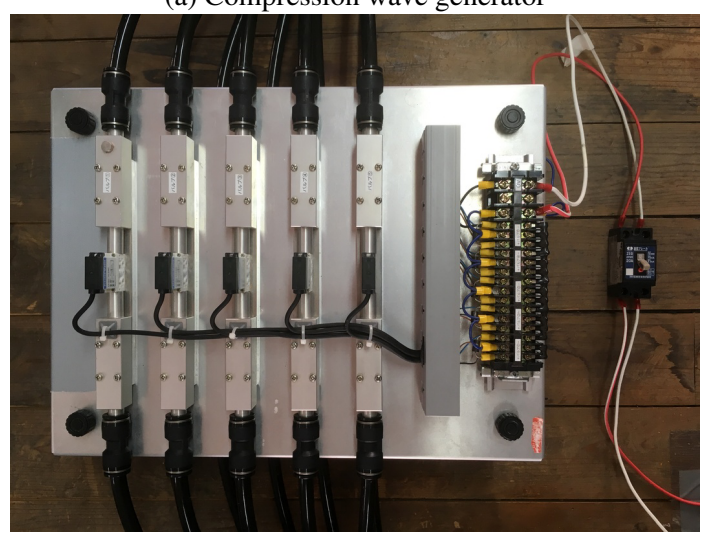

(b) Valves

Fig. 3 Photographs of the tunnel compression wave generator and valves

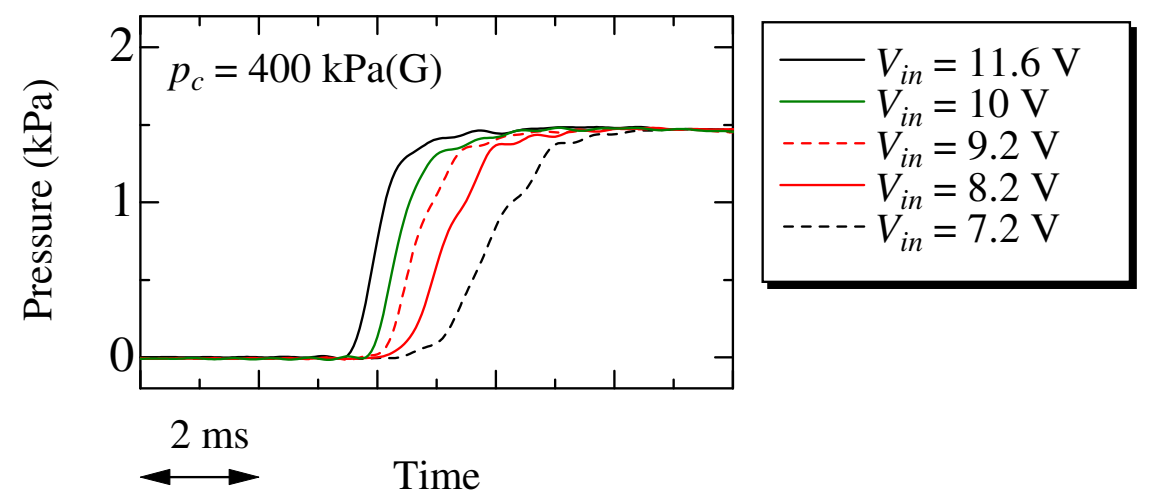

(a) $p_{c}=400 \mathrm{kPa}(\mathrm{G})$

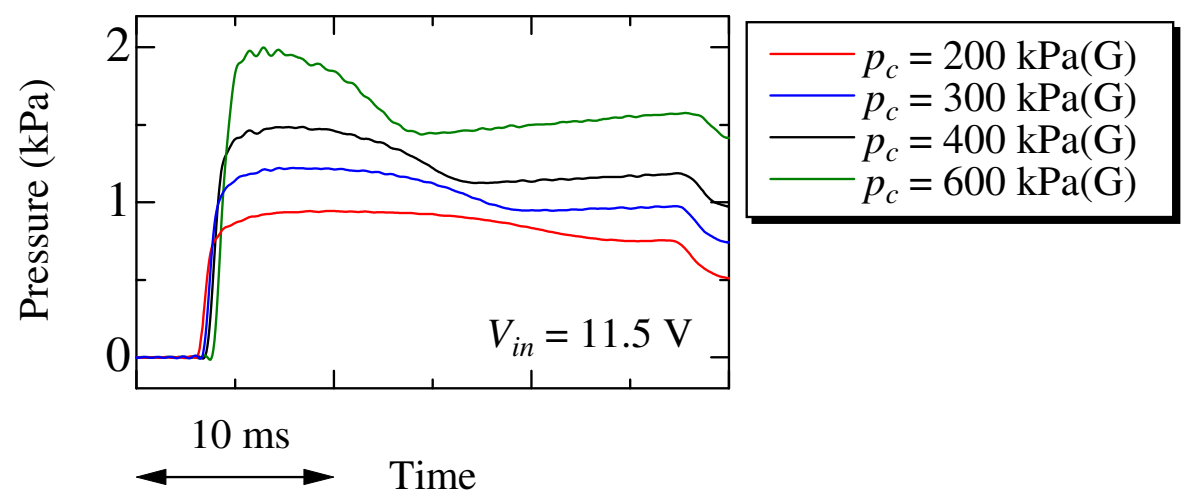

(b) $V_{\text {in }}=11.5 \mathrm{~V}$

Fig. 4 Compression waveforms 4 
and ZE2 for Fig. 5 (a) and XC1 and YC1 for Fig. (b). This time can be extended by extending $L_{t}$ and/or $L$. However, restrictions on this extension are imposed by factors such as the test room layout and effects of friction in long tubes.

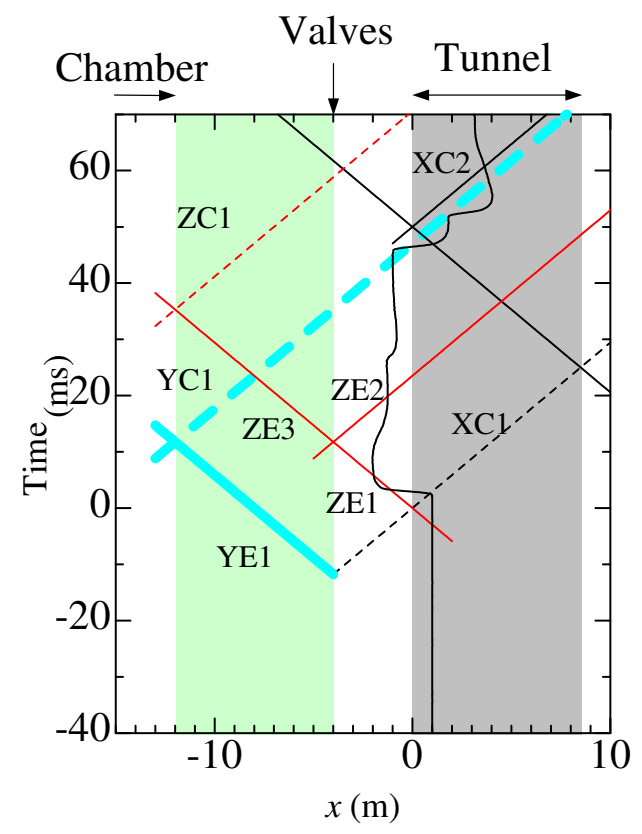

(a) $L_{c}=8 \mathrm{~m}, L_{t}=4 \mathrm{~m}$

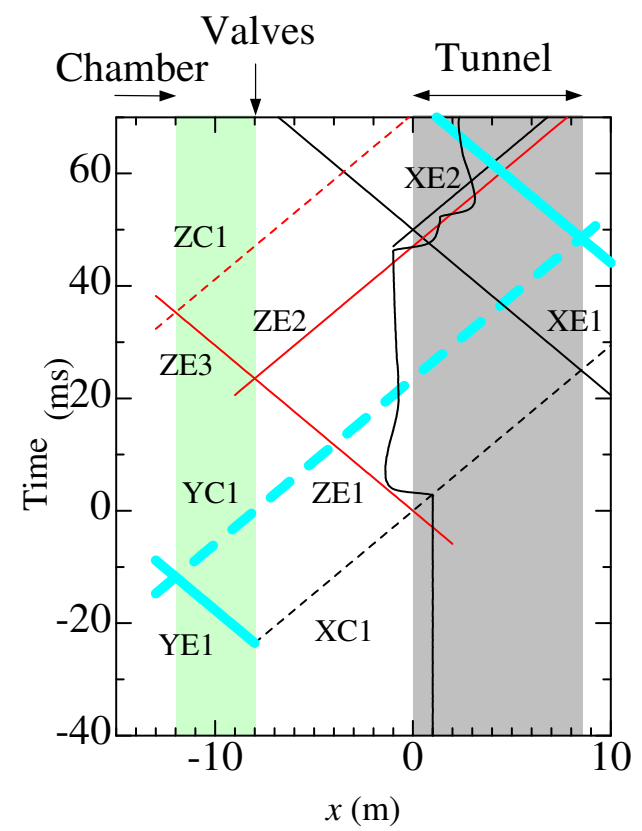

(b) $L_{c}=4 \mathrm{~m}, L_{t}=8 \mathrm{~m}$

Fig. $5 x-t$ diagrams for the different lengths of tubes between the valves, tunnel, and chamber (dotted lines: compression waves, solid lines: expansion waves)

\subsection{Relationship between $\Delta p$ and $p_{c}$}

This section describes the relationship between $\Delta p$ and $p_{c}$. Assuming that the temperature inside the chamber is constant at atmospheric temperature $T_{0}$, mass flow rate $m$ through the orifice in the solenoid valve is expressed as

$$
m=\sqrt{\gamma\left(\frac{2}{\gamma+1}\right)^{\frac{\gamma+1}{\gamma-1}} \frac{p_{c} A_{v}}{c_{0} / \sqrt{\gamma}}}
$$


where $\gamma$ is the specific heat ratio (= 1.4 for air) and $c_{0}$ is the speed of sound. Further, from the shock-wave equations, flow rate $m_{t}$ induced by the compression wave in the tubes is calculated as

$$
m_{t}=\rho_{t} u_{t} A_{t} \approx\left(1+\frac{3-\gamma}{4} \frac{\Delta p_{t}}{\gamma p_{0}}\right)^{\frac{1}{2}} \frac{\Delta p_{t}}{c_{0}} A_{t}
$$

where $\rho_{t}$ is the density behind the compression wave in the tubes, $u_{t}$ is the velocity induced by the compression wave in the tubes, and $A_{t}$ is the cross-sectional area of the tube connecting the valves and tunnel. By substituting Eq. (2) into Eq. (3), we have

$$
\frac{\Delta p_{t}}{p_{0}} \approx 1.75\left(-1+\sqrt{1+0.93 \frac{A_{v}}{A_{t}} \cdot \frac{p_{c}}{p_{0}}}\right)
$$

where $p_{0}$ is the atmospheric pressure. The relationship between the pressure in the tubes and tunnel model is obtained from the acoustic theory, such that

$$
\frac{\Delta p}{\Delta p_{t}} \approx \frac{2}{1+A /\left(n A_{t}\right)}
$$

where $n$ is the number of tubes. By substituting Eq. (4) into Eq. (5), we finally obtain

$$
\frac{\Delta p}{p_{0}} \approx \frac{3.5}{1+A /\left(n A_{t}\right)}\left(-1+\sqrt{1+0.93 \frac{A_{v}}{A_{t}} \cdot \frac{p_{c}}{p_{0}}}\right)
$$

As the compression wave attenuates because of friction during its propagation in the tubes and other pressure losses occur, Eq. (6) is modified to the following empirical form:

$$
\frac{\Delta p}{p_{0}} \approx \frac{3.5 k}{1+A /\left(n A_{t}\right)}\left(-1+\sqrt{1+0.93 \frac{A_{v}}{A_{t}} \cdot \frac{p_{c}}{p_{0}}}\right)
$$

where $k$ is an empirical parameter $(0<k<1)$. When determining the value of $k$ from experimental data, Eq. (7) gives an empirical formula of the relationship between $p_{c}$ and $\Delta p$. As Eq. (7) does not include temperature or density, $\Delta p$ can almost exactly be reproduced by only controlling $p_{c}$ as the change of $p_{0}$ is negligible. Figure 6 illustrates the waveforms of the compression waves generated by the TCG. Further, Fig. 7 shows the relationship between $p_{c}$ and $\Delta p$, and Fig. 8 shows the relationship between $V_{i n}$, pressure rise $\Delta p$, and maximum pressure gradient of the compression waves $(\partial p / \partial t)_{\max }$. The target values of the TCG are successfully achieved from Fig. 8. In the case of Fig. 7, $k=0.77$ yields a good agreement between Eq. (7) and the experimental data. Figure 6 also shows the waveform of a compression wave generated using a TLF in the RTRI by a train at a speed of $270 \mathrm{~km} / \mathrm{h}$ (red line). We can observe that the generator can simulate the tunnel compression wave generated by train entry. The generator is very effective because it produces a compression wave every 3 min at least, if needed, while the TLF produces a compression wave every 15 min. Furthermore, Fig. 6 also confirms that the valves open more quickly with a higher $V_{i n}$ value. In the case of small $V_{\text {in }}$, the pressure gradient waveforms have multiple peaks. When the solenoid valves are used below a certain voltage (limiting voltage, $V_{\text {lim }}$ ), the valves cannot open stably and they chatter because the solenoid valves are closed using chamber pressure $p_{c}$. Therefore, $V_{\text {lim }}$ depends on $p_{c}$; for example, $V_{\text {lim }}=9 \mathrm{~V}$ in the case of $p_{c}=400 \mathrm{kPa}(\mathrm{G})$ and $V_{\text {lim }}<7 \mathrm{~V}$ in the case of $p_{c}=200 \mathrm{kPa}(\mathrm{G})$.

As mentioned earlier, $\Delta p$ and $\Delta t$ of the compression waves are controlled by $p_{c}$ and $V_{i n}$, respectively. When a different relationship is required between $(\partial p / \partial t)_{\max }$ and the applied voltage (Fig. 8), we can also change $n$ to control $\Delta p$ and $(\partial p / \partial t)_{\max }$. Furthermore, when a multiple-peak pressure gradient waveform is required, such as those observed for tunnels with a hood (Ozawa (1979), Miyachi et al. (2016)), $L_{t}$ can be changed to $L_{t}+\Delta L$. For instance, let the length of half the tubes be $L_{t}+\Delta L$. In this case, two peaks are observed in the pressure gradient waveform of the generated compression wave. As the distance between the peaks is $\Delta L / c_{0}$, the estimation of the required $\Delta t$ is straightforward. However, the control of such multiple-peak pressure gradient waveforms is more difficult than the control of single-peak waveforms because it requires the control of many parameters.

\section{Comparison between TLF and TCG}

Table 1 summarises the comparison between a TLF and the TCG. The construction cost of a TLF is more than $500,000 \$$, and it would be higher if a special building is needed to install the facility. A TLF is usually operated by some 


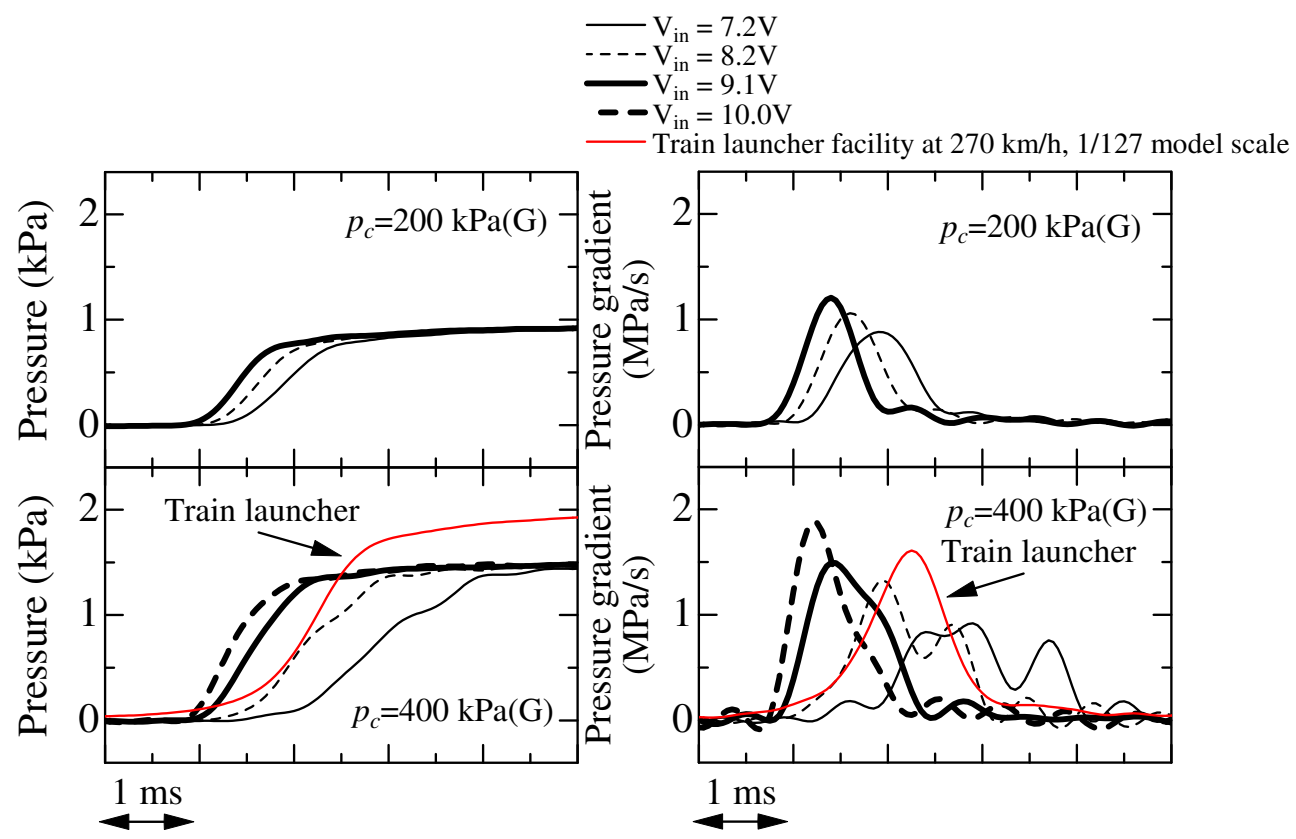

Fig. 6 Waveforms simulated using a tunnel compression wave generator

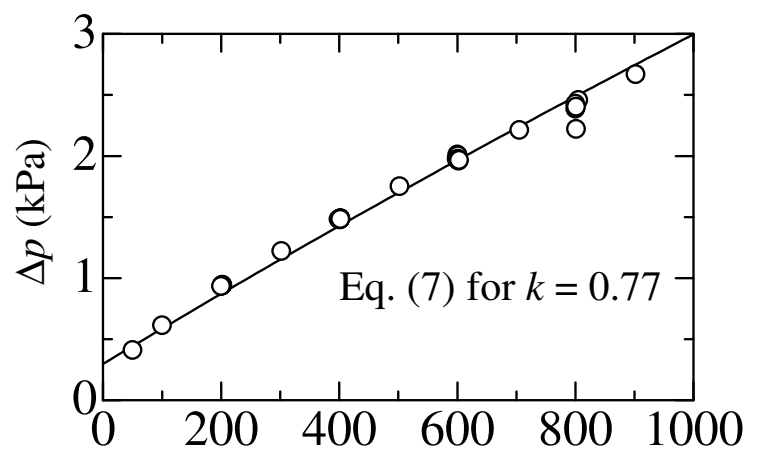

Chamber pressure $(\mathrm{kPa}(\mathrm{G}))$

Fig. 7 Relationship between pressure rise and chamber pressure 
$\circ p_{c}=200 \mathrm{kPa}(\mathrm{G})$

- $p_{c}=400 \mathrm{kPa}(\mathrm{G})$

$\square \quad p_{c}=600 \mathrm{kPa}(\mathrm{G})$

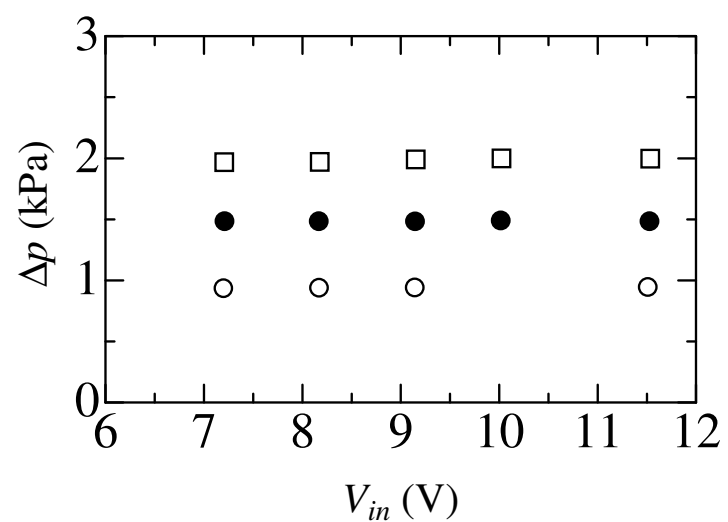

(a) Relationship between $\Delta p$ and $V_{\text {in }}$

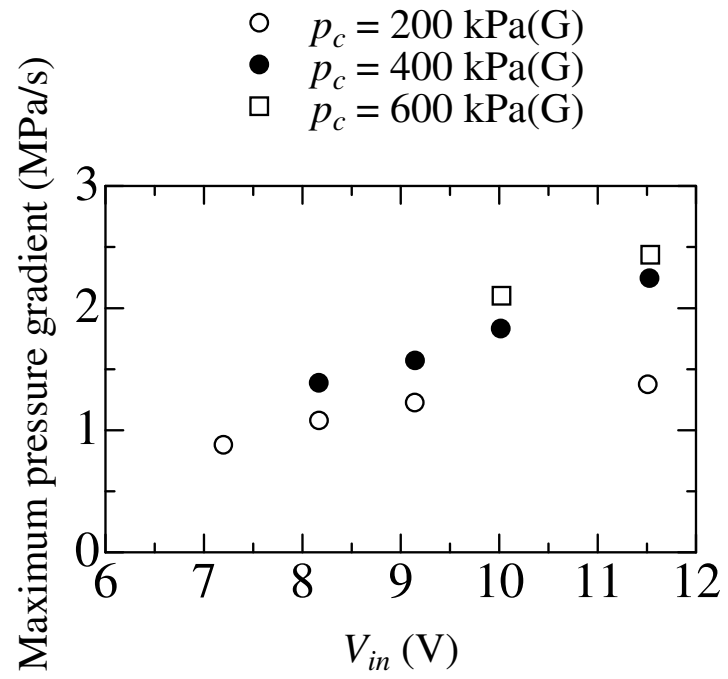

(b) Relationship between $(\partial p / \partial t)_{\max }$ and $V_{\text {in }}$

Fig. 8 Relationship among the pressure rise $\Delta p$, maximum pressure gradient $(\partial p / \partial t)_{\max }$, and voltage applied to valves $V_{\text {in }}$ 
persons and requires at least three procedures for each run: cleaning the train model, preparing the brake system, and resetting the train model at the launcher. Approximately $5 \mathrm{~min}$ are required for each procedure. Thus, the TLF can run at intervals of $15 \mathrm{~min}$.

As commercial products are used to build the TCG and it can easily be operated by only one person, its construction, operation, and maintenance costs are more affordable. All components of the TCG are compact, and thus, the TCG can be easily and quickly set up/dismantled, moved anywhere and placed in any free space only when used. In addition, tests can be conducted anywhere. Only the chamber of the TCG requires recharging and only for a few minutes as a very small volume of air is required in the chamber. Therefore, the data productivity of a TLF is lower than that of the TCG by $60 \%$, and the cost for each run of the TCG is significantly lower than that required for the TLF.

A TLF simulates all phenomena generated by a train in various environments including a tunnel, open air, and station, while the TCG can be used only for compression wave propagation in a tunnel and MPW emission. The TCG is more effective for phenomena expressed well through the linear acoustic theory, e.g., investigation of the influence of branches, resonators, absorbers, MPW emission, and topography of the ground, because the amplitude of pressure and wavelength are important for liner theory, and the influence of the detailed waveform can be easily calculated because of its linearity.

Although the benefits of the TCG include cost effectiveness and high data productivity, its usage is limited than that of TLFs. Our proposed TCG only generates a single-peak pressure gradient waveform at this stage, implying that the TCG can only simulate tunnel compression waves generated in tunnels without hoods. Today, as many tunnel portals have a hood, the TCG should be improved to easily generate and control multi-peak pressure gradient waveforms.

Table 1 Comparison between a train launcher facility and tunnel compression wave generator

\begin{tabular}{|c|c|c|}
\hline & Train launcher facility & $\begin{array}{c}\text { Tunnel compression } \\
\text { wave generator }\end{array}$ \\
\hline Order of construction cost (\$) & 500,000 & 10,000 \\
\hline Operation and maintenance cost & Expensive & Affordable \\
\hline Time for each run (min) & 15 & 3 \\
\hline Mobility & permanently fixed to a building & $\begin{array}{c}\text { easily moved anywhere, } \\
\text { quickly set up / } \\
\text { dismantled }\end{array}$ \\
\hline Decomposition or recomposition (day) & very difficult & 0.5 \\
\hline $\begin{array}{c}\text { Evaluation of train nose shape and tunnel } \\
\text { configuration }\end{array}$ & possible & impossible \\
\hline $\begin{array}{c}\text { Evaluation of compression wave } \\
\text { propagation and MPW emission }\end{array}$ & possible & impossible \\
\hline $\begin{array}{c}\text { Evaluation of other phenomena generated } \\
\text { by a train }\end{array}$ & possible \\
\hline
\end{tabular}

\section{Conclusion}

An affordable TCG was developed in this study. The designed generator consists of a pressure chamber, a compressor, multiple valves, a power supply system, and a tunnel model. It simulates the tunnel compression wave generated by a high-speed train entering a tunnel. As the effective cross-sectional areas of the high-speed solenoid valves are insufficient, we employed multiple valves that can be opened simultaneously. As we used commercial and affordable valves for the generator, the cost and time for developing the generator were reduced. This generator is very compact and has high data productivity compared to TLFs.

The following conclusions were drawn regarding the performance of the generator.

( 1 ) The TCG provides compression waves with pressure rises and time wavelengths that are almost identical to those of the tunnel compression waves generated by TLFs without tunnel hoods.

( 2 ) The pressure rise of a compression wave is controlled by changing the chamber pressure. An empirical formula is introduced to estimate the relationship between the compression wave pressure rise and chamber pressure.

(3) The time wavelength of the compression waves is controlled by changing the voltage applied to the valves.

A TLF simulates not only the influence of the train nose but also various phenomena that are generated by entry and passage of the train through a tunnel. In contrast, a TCG easily facilitates small-element experiments. Thus, it is necessary to utilise these facilities appropriately according to the test requirements and aims. The generator is expected 
to provide sufficient data to develop measures against MPWs in the stages of propagation and emission as the generator can produce a compression wave very quickly. In the next stage, experimental data from the TCG for the propagation of tunnel compression waves and/or the emission of MPWs should be compared with data obtained using theoretical and numerical methods.

\section{Acknowledgment}

The authors would like to thank Prof. Toshiyuki Aoki of Kyusyu University, Japan, for the technical advice and in-depth discussions on the tunnel compression wave generator. We also thank KOGANEI Inc. for their technical support on solenoid valves.

\section{References}

Fukuda, T., Experimental rig for the micro-pressure wave (in Japanese), Journal of INCE/J, Vol. 37 (2013), pp. 90-95.

Hara, T., Aerodynamic force acting on a high speed train at the tunnel entrance, Bulletin of JSME, Vol. 4 (1961), pp. 547-553.

Heine, D. and Ehrenfried, K., Experimental study of the entry of a high-speed train into a railway tunnel, International Journal of Railway Technology, Vol. 1 (2012), pp. 1-18.

Iida, M., Matsumura, T., Fukuda, T., Nakatani, K. and Maeda, T., Optimization of train nose shape for reducing impulsive pressure wave from tunnel exit, Transactions of the Japan Society of Mechanical Engineers, Series B, Vol. 62 (1996), pp. 1428-1435. (in Japanese)

Johnson T. and Dalley, S., 1/25 scale moving model tests for the TRANSAERO project, Notes on numerical fluid mechanics and multidisciplinary design, Vol. 79 (2002), pp. 123-135.

Martin, W. A., A review of shock tubes and shock tunnels, CONVAIR. ASTRONAUTICS., Report No. ZR-658-050, 1959.

Matsuo, T., Aoki, T., Kashimura, H. and Takeuchi, M., Attenuation of compression waves in a high-speed railway tunnel simulator, Proceedings of 7th International Conference on Aerodynamics and Ventilation of Vehicle Tunnel (1991), pp. 239-253.

Miyachi, T. and Fukuda, T., Experimental investigation of the effects of topography around the tunnel portal on micropressure waves, Quarterly Report of RTRI, Vol. 55 (2014), pp. 235-240.

Miyachi, T., Fukuda, T. and Saito, S. Model experiment and analysis of pressure waves emitted from portals of a tunnel with a branch, Journal of Sound and Vibration, Vol. 333 (2014), pp. 6156-6169.

Miyachi, T., Acoustic model of micro-pressure wave emission from a high-speed train tunnel, Journal of Sound and Vibration, Vol. 391 (2016), pp. 127-152.

Miyachi, T., Saito, S., Fukuda, T., Sakuma, Y., Ozawa, S., Arai, T., Sakaue, S. and Nakamura, S., Propagation characteristics of tunnel compression waves with multiple peaks in the waveform of the pressure gradient: Part 1: Field measurements and mathematical model, Proceedings of the Institution of Mechanical Engineers, Part F: Journal of Rail and Rapid Transit, Vol. 230 (2016), pp. 1297-1308.

Ozawa, S., Studies of micro-pressure wave radiated from a tunnel exit (in Japanese), Railway Technical Research Report, No. 1121, 1979.

Ozawa, S., Murata, K. and Maeda, T., Effect of ballasted track on distortion of pressure wave in tunnel and emission of micro-pressure wave, Proceedings of 9th International Conference on Aerodynamics and Ventilation of Vehicle Tunnels (1997), pp. 935-950.

Sugimoto, N., Masuda, M., Yamashita, K. and Horimoto, H., Verification of acoustic solitary waves, Journal of Fluid Mechanics, Vol. 504 (2004), pp. 271-299.

Tebbutt, J., Vahdati, M., Carolan, D. and Dear, J., Numerical investigation on an array of Helmholtz resonators for the reduction of micro-pressure waves in modern and future high-speed rail tunnel systems, Journal of Sound and Vibration, Vol. 400 (2017), pp. 606-625.

Vardy, A. and Brown, J., Influence of ballast on wave steepening in tunnels, Journal of Sound and Vibration, Vol. 238 (2000), pp. 595-615.

Yamamoto, A. Micro-pressure wave radiated from tunnel exit (in Japanese), Preprint of the spring Meeting of physical society of Japan, 4p-H-4 (1977), p. 137. 
Yang, Q.-S., Song, J.-H. and Yang, G.-W., A moving model rig with a scale ratio of 1/8 for high speed train aerodynamics, Journal of Wind Engineering and Industrial Aerodynamics, Vol. 152 (2016), pp. 50 - 58.

Zhou, D., Tian, H.-Q., Zhang, J. and Yang, M.-Z., Pressure transients induced by a high-speed train passing through a station, Journal of Wind Engineering and Industrial Aerodynamics, Vol. 135 (2014), pp. 1 - 9. 